\title{
The role of Ureaplasma parvum serovar-3 or serovar-14 infection in female patients with chronic micturition urethral pain and recurrent microscopic hematuria
}

\author{
Moon Seong Kim ${ }^{1}$, Dong Hyun Lee ${ }^{2}$, Tae Jin Kim ${ }^{1}$, Jong Jin $\mathrm{Oh}^{3}$, Seung Ryeol Rhee ${ }^{1}$, Dong Soo Park ${ }^{1}$, \\ Young Dong $\mathbf{Y u}^{1 \wedge}$ \\ ${ }^{1}$ Department of Urology, CHA Medical University, College of Medicine, Bundang CHA Hospital, Seongnam, Korea; ${ }^{2}$ Department of Physiology, \\ CHA Medical University, College of Medicine, Seongnam, Korea; ${ }^{3}$ Department of Urology, Seoul National University, College of Medicine, Seoul \\ National University Bundang Hospital, Seoul, Korea \\ Contributions: (I) Conception and design: MS Kim, YD Yu; (II) Administrative support: TJ Kim, YD Yu; (III) Provision of study materials or patients: \\ TJ Kim, JJ Oh, SR Rhee, DS Park; (IV) Collection and assembly of data: MS Kim, TJ Kim, JJ Oh, SR Rhee, DS Park; (V) Data analysis and \\ interpretation: MS Kim, TJ Kim, JJ Oh, SR Rhee, DS Park; (VI) Manuscript writing: All authors; (VII) Final approval of manuscript: All authors. \\ Correspondence to: Young Dong Yu, MD. Department of Urology, CHA Medical University, College of Medicine, Bundang CHA Hospital,9 Yatap-ro \\ Bundang-gu, Seongnam-siGyeonggi-do,13496, Korea. Email: danielyu0714@gmail.com.
}

Background: Ureaplasma parvum (UP) is commonly isolated in the genitor-urinary tract and may cause various clinical features, including microscopic hematuria $(\mathrm{MH})$. Some UP serovars are more commonly related with specific urogenital disease, but the evidences have been conflicting. This study primarily aimed to research the possible associations between specific UP serotypes and genito-urinary pathogenicity in female patients showing MH with/without chronic micturition urethral pain (CMP).

Methods: This study retrospectively reviewed 276 female patients having MH with/without CMP, who visited health screening center or female infertility clinic. All patients underwent multiplex polymerase chain reaction (PCR) tests with vaginal and urine samples to evaluate the infection rate and serotypes of UP. The antimicrobial susceptibility of UP and the predictors of CMP among UP infected patients were also analyzed. All patients were followed up at least for 6-months.

Results: Forty-nine patients (17.8\%) showed urinary UP infection. Urinary UP serotyping showed the prevalence of seorvar-1, -3 , -6 and -14 were $24.5 \%, 30.6 \%, 18.4 \%$ and $26.5 \%$, respectively. $79.6 \%$ of the urinary UP positive patients accompanied vaginal UP infection. 22 patients of the cohort (8.0\%) had CMP whereas serovars-3 and -14 accompanied CMP in 54.5\% and $41.0 \%$ cases, respectively. No serovars-6 infection case had CMP. $26.4 \%$ of the cohort were infertile whereas $10.9 \%$ of these infertile patients were positive for urinary tract infection with UP serotype-3 or -14 . Doxycycline, josamycin and pristinamycin were the most active antibiotics with the lowest rate of resistance $(0.0 \%)$ for treating UP. At 1 -month postinitial treatment with doxycycline, all UP serotypes were eradicated and no patient complained of urethral discomfort. However, simultaneous urinary and vaginal reinfection of serovar-3 (5 cases) and serovar-5 (1 case) were confirmed at 3-months post-initial doxycycline therapy. The logistic regression analyses revealed that serovars-3 [hazard ratio (HR) 1.354, P value 0.018 ] and -14 (HR 1.103, P value 0.046) were significantly associated with CMP in female patients having $\mathrm{MH}$.

Conclusions: UP serovars-3 and -14 infections could be associated with CMP in female patients having MH. Doxycycline, josamycin and pristinamycin were effective for treating UP. Serovar-3 showed higher reinfection rate than other serotypes after antibiotics treatment.

Keywords: Ureaplasma parvum (UP); serotype; microscopic hematuria

\footnotetext{
$\wedge$ ORCID: 0000-0002-2696-7004.
} 
Submitted May 10, 2020. Accepted for publication Sep 18, 2020.

doi: $10.21037 /$ tau-20-920

View this article at: http://dx.doi.org/10.21037/tau-20-920

\section{Introduction}

Urinalysis is commonly included in routine health screening tests as it is relatively cheap and handy modality for detecting incidental microscopic hematuria $(\mathrm{MH})$, where the prevalence of $\mathrm{MH}$ ranges from $2 \%$ to $31 \%(1,2)$. Although, the clinical significance of $\mathrm{MH}$ is debatable, $\mathrm{MH}$ can be an initial sign of the urinary tract malignancy whereas urologic malignancies are observed in almost $5 \%$ of patients with $\mathrm{MH}$ (3). $\mathrm{MH}$ also has been regarded as a non-significant predictor of long-term renal function in the patients with chronic kidney disease (CKD) $(4,5)$.

Ureaplasma and mycoplasma are pleomorphic, cell wall deficient microorganisms, which are commonly isolated in the genitourinary tract of asymptomatic healthy subjects and also in the people with lower urinary tract symptoms (LUTS) and other various clinical features, including $\mathrm{MH}$ $(6,7)$. Ureaplasma species consists of 2 biovars, Ureaplasma parvum (UP) and Ureaplasma urealyticum (UU), and 14 serovars (serotypes) whereas the sub-classification is dependent on the differences of specific genetic sequences such as16S rRNA gene sequences, urease gene, and the multiple-banded antigen (MBA) genes. Among Ureaplasma serovars, 4 serovars (serovars-1, $-3,-6$ and -14 ) belong to UP, while UU comprises the other serovars (serovars-2, $-4,-5,-7$ and from -7 to -13$)(8,9)$. The association of UP with medical therapy needed urinary tract infections (UTI) is still controversial and underestimated, while the pathogenic role of UU with urogenital tract infections is relatively well established (10). However, some previous studies including Kacerovsky et al. (11) have suggested the clinical significance of UP by describing the prevalence of UP was $57 \%$ in healthy non-pregnant women, which makes UP distinctively more prevalent than any of the other urogenital mycoplasma species. It has been reported that some UP serovars are more commonly related with specific urogenital disease than other serovars or normal flora, but the evidences have been limited and conflicting due to the technical difficulties of conventional genotyping methods $(12,13)$. In addition, some researchers reported that only serovars-3, but neither serovars- 1 nor serovars-6, is associated with preterm delivery at early gestational age in pregnant women positive for vaginal UP colonization (14).
To our knowledge, few studies have investigated the UP serovars specific urinary pathogenicity, including chronic micturition urethral pain (CMP) and MH. Therefore, this study primarily aimed to research the possible associations between specific UP serovars and genitourinary pathogenicity, including persistent UTI and infertility, in female patients showing $\mathrm{MH}$ with or without CMP by performing polymerase chain reaction (PCR)based serotyping. Secondarily, the current study analyzed the antibiotic sensitivity according to different UP serovars. We present the following article in accordance with the STROBE reporting checklist (available at http://dx.doi. org/10.21037/tau-20-920).

\section{Methods}

\section{Patient selection and clinical parameters}

The study was a single center retrospectively designed cohort study of female patients who were referred to urology clinic between January 2018 to March 2019 because of $\mathrm{MH}$ confirmed during their visit to health screening center or female infertility clinic for the purpose of regular health checkup or fertility examination, respectively. All procedures performed in this study were in accordance with the Declaration of Helsinki (as revised in 2013) and approved by the Ethics Committee of the Bundang CHA Hospital, CHA Medical University (registration ID 202005-016). All patients enrolled in the study completed the written informed consent form regarding use of their medical records. Clinical data collection and all medical procedures were undertaken according to relevant guidelines and regulations. The clinical parameters of the study subjects such as age, presence of micturition urethral pain, estimated glomerular filtration rate (eGFR), presence of proteinuria, $\mathrm{MH}$ grade, urine cytology, underlying diseases, prevalence of infertility, presence of urine and vaginal Ureaplasma, and history UTI were reviewed (Table 1). Regarding micturition urethral pain, all study subjects were inquired whether they had experienced any discomfort felt in urethra during urination, and voiding urethral discomfort lasted more than 3-months was defined as CMP in this study. Abdomino-pelvis computed tomography (CT) was 
Table 1 Baseline characteristics and UP infection related clinical factors $(\mathrm{n}=276)$

\begin{tabular}{|c|c|}
\hline Clinical factors & Values \\
\hline \multicolumn{2}{|l|}{ A. Basic characteristic } \\
\hline Age (years), mean $\pm \mathrm{SD}$, (range) & $42.5 \pm 11.3(21-68)$ \\
\hline \multicolumn{2}{|l|}{ Age group (years), n (\%) } \\
\hline $20-30$ & $67(24.3)$ \\
\hline $31-40$ & $64(23.2)$ \\
\hline $41-50$ & $76(27.5)$ \\
\hline $51-60$ & $69(25.0)$ \\
\hline eGFR $\left(\mathrm{mL} / \mathrm{min} / 1.73 \mathrm{~m}^{2}\right)$, mean $\pm \mathrm{SD}$ & $113.7 \pm 5.6$ \\
\hline Presence of proteinuria, n (\%) & $0(0.0)$ \\
\hline Hemoglobin $(\mathrm{g} / \mathrm{dL})$, mean $\pm \mathrm{SD}$ & $11.9 \pm 3.3$ \\
\hline \multicolumn{2}{|l|}{ §Urine cytology, n (\%) } \\
\hline Negative cytology & 248 (89.9) \\
\hline Atypical cells present & $0(0.0)$ \\
\hline Neutrophils present & $26(9.4)$ \\
\hline \multicolumn{2}{|c|}{ Microscopic hematuria grade, RBCs/HPF, n (\%) } \\
\hline $0-3$ & $0(0.0)$ \\
\hline $4-10$ & $83(30.1)$ \\
\hline $11-25$ & $172(62.3)$ \\
\hline $26-50$ & $21(7.6)$ \\
\hline \multicolumn{2}{|l|}{ Underlying disease, n (\%) } \\
\hline Hypertension & $102(36.9)$ \\
\hline Angina pectoris & $15(5.4)$ \\
\hline DM & $64(23.2)$ \\
\hline Cerebral infarction & $8(2.9)$ \\
\hline Anti-coagulant medication, $\mathrm{n}(\%)$ & $0(0.0)$ \\
\hline Anti-hypertensive medication, $\mathrm{n}(\%)$ & $117(42.4)$ \\
\hline \multicolumn{2}{|c|}{$\begin{array}{l}\text { B. UP infection rate, urinary tract op. } \mathrm{Hx} \text {. and other related } \\
\text { urogenital symptoms }\end{array}$} \\
\hline Urinary tract UP infection, $\mathrm{n}(\%)$ & 49/276 (17.8) \\
\hline Serovar-1 & $12 / 49(24.5)$ \\
\hline Serovar-3 & $15 / 49(30.6)$ \\
\hline Serovar-6 & 9/49 (18.4) \\
\hline Serovar-14 & 13/49 (26.5) \\
\hline $\begin{array}{l}\text { Co-presence of pyuria (neutrophils) } \\
\text { among urinary UP infection }\end{array}$ & $26 / 49(53.1)$ \\
\hline
\end{tabular}

Table 1 (continued)
Table 1 (continued)

\begin{tabular}{|c|c|}
\hline Clinical factors & Values \\
\hline Serovar-1 & $4 / 26(15.4)$ \\
\hline Serovar-3 & $11 / 26(42.3)$ \\
\hline Serovar-6 & $2 / 26(7.7)$ \\
\hline Serovar-14 & 9/26 (34.6) \\
\hline $\begin{array}{l}\text { Vaginal UP infection among urinary tract } \\
\text { UP infections, } n(\%)\end{array}$ & $39 / 49(79.6)$ \\
\hline Serovar-1 & 7/39 (17.9) \\
\hline${ }^{9}$ Vaginal discharge present & $1 / 7(14.3)$ \\
\hline Serovar-3 & $15 / 39(38.5)$ \\
\hline "Vaginal discharge present & $7 / 15(46.7)$ \\
\hline Serovar-6 & 4/39 (10.2) \\
\hline "Vaginal discharge present & $2 / 4(50.0)$ \\
\hline Serovar-14 & $13 / 39(33.4)$ \\
\hline "Vaginal discharge present & $8 / 13(61.5)$ \\
\hline \multicolumn{2}{|l|}{ Hx. of previous urinary tract op. $\mathrm{n}(\%)$} \\
\hline Present & $0(0.0)$ \\
\hline Absent & $276(100.0)$ \\
\hline \multicolumn{2}{|l|}{$\begin{array}{l}{ }^{\circ} \text { Co-infection of genitourinary tract with } \\
\text { other infectious entities }\end{array}$} \\
\hline Bacterial vaginosis & $0(0.0)$ \\
\hline UTI & $0(0.0)$ \\
\hline
\end{tabular}

C. UP infection, female infertility, UP infection $\mathrm{Hx}$ and $\mathrm{Hx}$ of urinary tract surgery

${ }^{\mp}$ Chronic micturition urethral pain, $\mathrm{n}(\%)$

Present

$22 / 276(8.0)$

Serovar-1

$1 / 22(4.5)$

Serovar-3

$11 / 22(50.0)$

Serovar-6

$1 / 22(4.5)$

Serovar-14

$9 / 22(41.0)$

Absent

$254 / 276(92.0)$

Infertility rate

Fertile

$203 / 276(73.6)$

Infertile

$73 / 276$ (26.4)

Ovulatory factor infertility

$14 / 73(19.2)$

Tubaric factor infertility

$47 / 73(64.4)$

Table 1 (continued) 
Table 1 (continued)

\begin{tabular}{|c|c|}
\hline Clinical factors & Values \\
\hline $\begin{array}{l}\text { Uterine factor infertility (endometriosis, } \\
\text { uterine synechiae) }\end{array}$ & $39 / 73(53.4)$ \\
\hline $\begin{array}{l}\text { Urinary tract UP infection among infertile } \\
\text { patients, } \mathrm{n}(\%)\end{array}$ & $8 / 73(10.9)$ \\
\hline Serovar-1 & $0 / 8(0.0)$ \\
\hline Serovar-3 & 6/8 (58.8) \\
\hline Ovulatory factor infertility & $0 / 6(0.0)$ \\
\hline Tubaric factor infertility & $5 / 6(83.3)$ \\
\hline Uterine factor infertility & $1 / 6(16.7)$ \\
\hline Serovar-6 & $0 / 8(0.0)$ \\
\hline Serovar-14 & $2 / 8(25.0)$ \\
\hline Ovulatory factor infertility & $0 / 2(0.0)$ \\
\hline Tubaric factor infertility & $2 / 2(100.0)$ \\
\hline Uterine factor infertility & $0 / 2(0.0)$ \\
\hline $\begin{array}{l}\text { Hx of previous urinary/vaginal UP } \\
\text { infection, } n(\%)\end{array}$ & $9(3.3) / 21(7.6)$ \\
\hline
\end{tabular}

$\mathrm{SD}$, standard deviation; eGFR, estimated glomerular filtration rate; RBCs, red blood cells; HPF, high power filed; DM, diabetes mellitus; UP, Ureaplasma parvum; op., operation; $\mathrm{Hx}$, history; UTI, urinary tract infection. ${ }^{\S}$ Serovar-3/-14 infected patients were present with neutrophils in urine. "Vaginal discharge accompanied by vaginal UP infection showed whitish or clear characteristics. ${ }^{\text {TO}}$ Only serovar-3/-14 infected patients showed both chronic voiding urethral pain and microscopic hematuria. The intensity of voiding pain was mild (NRS $\leq 3)$ in all patients who complained of micturitional discomfort. "Bacteria vaginosis and UTI were initially screened by multiplex realtime polymerase chain reaction (PCR) of vaginal swab and urine samples detecting 12 pathogens including Chlamydia trachomatis, Neisseria gonorrhoeae, Mycoplasma hominis, Trichomonas vaginalis, Ureaplasma urealyticum, Mycoplasma genitalium, Herpes simplex virus-1 and -2, Gardnerella vaginalis, Treponema pallidum, Candida albicans, and Ureaplasma parvum.

also performed to all study subjects to detect the presence of urinary stones or other possible causes of CMP. During the data collection period, 521study subjects with $\mathrm{MH}$ were initially analyzed. Among them, the patients who had received antibiotics therapy due to urogenital infection during the recent six months were removed from the final investigation to minimize bias affecting the antibiotic susceptibility analyses of Ureaplasma species. The patients who were on anticoagulant medications were also excluded from the study as it could be a potential risk factor of $\mathrm{MH}$. In addition, the patients with urinary stone or previous history of urologic surgery were excluded from the study. Anyone tested positive for UTI or vaginal infection with pathogens other than UP according to multiplex PCR tests were also excluded from the analysis. As this study focused on evaluating the specific UP serovars type that might be associated with genitourinary infection accompanying $\mathrm{MH}$ and CMP, the patients confirmed with only vaginal UP infection, but not urinary infection were excluded from the study. After removing those subjects who met the exclusion criteria, overall 276 female patients with $\mathrm{MH}$ (age: 23-58) were included in the final analysis (Figure 1).

\section{Specimen collection and culture}

Urine sample and vaginal swab sample were collected from all 276 patients. Urine samples were collected by clean urethral catheterization. After introducing a sterile speculum into the vagina, excessive vaginal and cervical secretions were removed by a sterile cotton swab, which was followed by vaginal swab sample collection from the cervical transitional zone. No antiseptic solutions were used during the specimen collections. Both urine and vaginal sample collecting procedures were performed cautiously to prevent hemorrhage from the target organs such as urethra or uterine cervix. All clinical samples were collected twice. The first samples were used for analyzing the presence of possible genitourinary pathogen by performing multiplex polymerase chain reaction (PCR) tests. The second samples were used for additional confirmation and serotyping of UP, which was undertaken by using sets of real time multiplex PCR. To verify recurrent genitourinary UP infection, urine and vaginal samples from all study cohort were repeatedly collected at 1-, 3-and 6-months after initial analysis.

\section{Molecular characterization of genitourinary pathogens}

The first urine and vaginal swab samples collected from the cohort were tested for any genitourinary pathogens. QIAamp DNA Mini Kit (Qiagen, Germany) was used for DNA extraction from acquired specimens, which was undertaken according to the instructions provided by the manufacturer. Afterward, a multiplex real-time PCR assay DiaPlexQ ${ }^{\text {TM }}$ STI 12 Detection Kit (Solgent Co., Ltd., South Korea) was used for to detect 12 different sexually transmitted infection (STI) pathogens including Chlamydia trachomatis (CT), Neisseria gonorrhoeae (NG), 


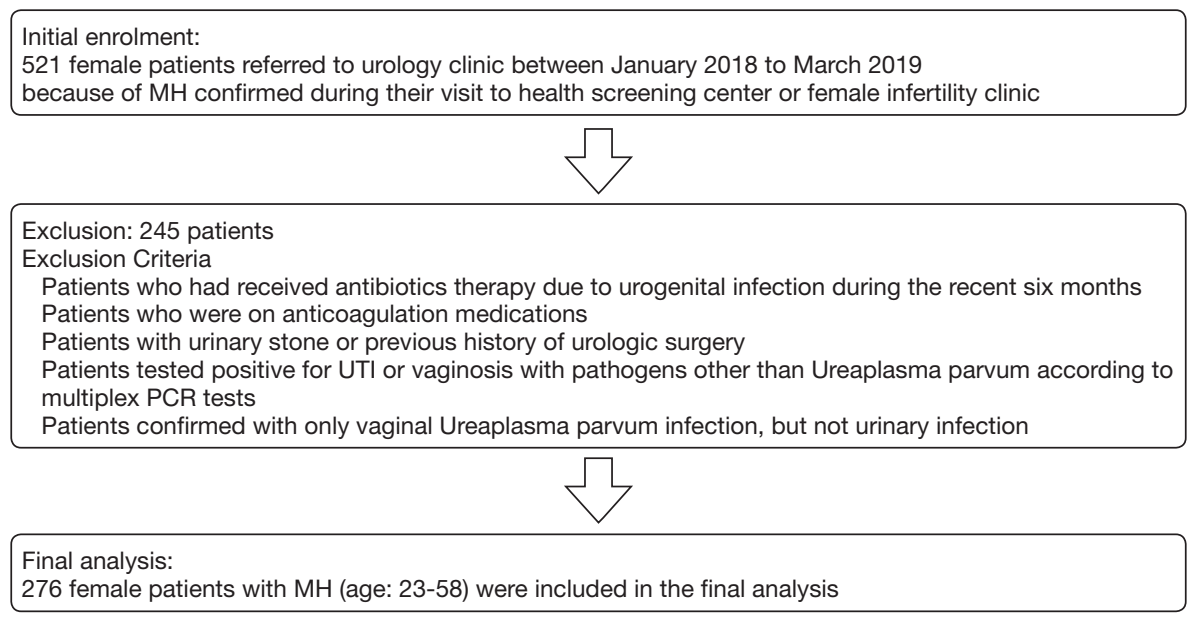

Figure 1 Consort diagram showing the exclusion criteria and the number of patients who were included in the analysis. MH, microscopic hematuria; UTI, urinary tract infection; PCR, polymerase chain reaction.

Mycoplasma hominis (MHO), Trichomonas vaginalis (TV), Ureaplasma urealyticum (UU), Mycoplasma genitalium (MG), Herpes simplex virus (HSV)-1 and -2, Gardnerella vaginalis (GV), Treponema pallidum (TP), Candida albicans (CA) and UP. $15 \mu \mathrm{L}$ PCR premix tubes, which initially contained $5 \mu \mathrm{L}$ of Primer/Probe Mixture and $10 \mu \mathrm{L}$ of Multiplex Real-Time PCR Smart mix, was loaded with the DNA extracted from specimens. PCR was performed by applying the 7500 Fast Real-Time PCR System (Applied Biosystems, USA). After incubation of the PCR mixture at $50{ }^{\circ} \mathrm{C}$ for 3 minutes, pre-denaturation (at $95^{\circ} \mathrm{C}$ for 15 minutes) and 45 cycles of PCR (at $95^{\circ} \mathrm{C}$ for 20 seconds followed by $60^{\circ} \mathrm{C}$ for 40 seconds) were sequentially undertaken. Cycle threshold (CT) $\leq 43$ for each target was considered as a positive result. This multiplex realtime PCR assay DiaPlexQ ${ }^{\text {TM }}$ STI 12 Detection Kit based genitourinary infection analysis was performed only once for each patient and no repeated tests were undertaken after the initial analysis.

\section{Confirmation and serotyping of UP}

The second urine and vaginal swab samples were used for additional confirmation and serotyping UP. The extraction of UP DNA from specimens was undertaken by using Reagents Genomics DNA Kit (Geneaid, USA). Conventionally available master mix kits (BioNeer, South Korea) was used for additional PCR confirmation of UP. The primers UMS-57 and UMA-222 were specifically targeted for the conserved region within 5' end of MBA gene (Table 2). 10 pmol of each primer was added to $20 \mu \mathrm{L}$ reaction mixture tubes. PCR mixture underwent the following steps; pre-denaturation (at $95{ }^{\circ} \mathrm{C}$ for 5 minutes), final denaturation (at $95{ }^{\circ} \mathrm{C}$ for 30 seconds), annealing (at $58^{\circ} \mathrm{C}$ for 30 seconds), 40 cycles of extension (at $72{ }^{\circ} \mathrm{C}$ for 1 minute), and final extension (at $72^{\circ} \mathrm{C}$ for 5 minutes). For serotyping of UP, the primers used (BioNeer, South Korea) were as follows; UMS-83/UMA-1A for serovar-1, UMS-3/ UMA-269 for serovar-3, UMS-54/UMA-269 for serovar-6, and UMS-14/UMA-314A for serovar-14 (Table 2). Serotyping of UP was performed according to the manufacturer's instruction as follows; initial denaturation (at $95{ }^{\circ} \mathrm{C}$ for 5 minutes), second denaturation (at $95{ }^{\circ} \mathrm{C}$ for 30 seconds), annealing (at $58{ }^{\circ} \mathrm{C}$ for 30 seconds), and 40 cycles of extension (at $72{ }^{\circ} \mathrm{C}$ for 1 minute with 40 cycles). The products obtained from the PCR procedures were applied to electrophoresis on $2 \%$ agarose gels with $0.5 \mathrm{mg} / \mathrm{mL}$ of ethidium bromide staining. Positive results were defined as a band with proper bandwidth visible on UV trans-illumination light. To verify the specific UP serotypes associated with recurrent genitourinary infection and $\mathrm{MH}$, these confirmation and serotyping of UP by using multiplex PCR were repeatedly performed to all patients at 1-, 3- and 6-month after initial analysis.

\section{Antibiotic susceptibility of UP}

A conventional available Mycoplasma IST-2 Kit (BioMerieux, France) was used to evaluate the antibiotic susceptibility of UP. This standardized kit analyzes 
Table 2 Target gene and PCR primers used for verification of UP and UP serovars

\begin{tabular}{|c|c|c|c|}
\hline Ureaplasma & Target gene & Primer & Sequence \\
\hline \multicolumn{4}{|c|}{ A. PCR primer used for the detection of UP infection } \\
\hline \multirow[t]{2}{*}{ UP } & MBA (5' ends) & UMS-57 & 5'-TAAATCTTAGTGTTCATATTTTTTAC-3' \\
\hline & & UMA-222 & 5'-GTAAGTGCAGCATTAAATTCAATG-3' \\
\hline \multicolumn{4}{|c|}{ B. PCR primer used for the detection of different UP serovars } \\
\hline Serovar-1 & & UMA-1A & 5'-TTTCTTITGGTTCTTCAGTTTTTGAAG-3' \\
\hline \multirow[t]{2}{*}{ Serovar-3 } & MBA & UMS-3 & 5'-TTACTGTAGAAATTATGTAAGATTACC-3' \\
\hline & & UMA-269 & 5'-CCAAATGACCTTTTGTAACTAGAT-3' \\
\hline Serovar-6 & MBA & UMS-54 & 5'-AATCTTAGTGTTCATATTIITTACTAG-3' \\
\hline Serovar-14 & & UMA-314A & 5'-GTTGTTCTTTACCTGGTTGTGTAG-3' \\
\hline
\end{tabular}

PCR, polymerase chain reaction; UP, Ureaplasma parvum, MBA, multiple-banded antigen.

the sensitivity to nine different antibiotics in two concentrations. This kit shows color changes when the $\mathrm{pH}$ level elevates because of the pathogens present on a liquid culture medium. The antibiotics used for susceptibility analysis were as follows; azithromycin, clarithromycin, josamycin, pristinamycin, erythromycin, tetracycline, doxycycline, ciprofloxacin, and ofloxacin.

According to the sensitivity interpretation guideline supplied by the manufacturer, the sensitivity was defined by color changes observed in different antibiotics concentrations. Antibiotic resistance was defined as the color alteration presented in both antibiotic concentrations whereas the color alteration presented only in the lowest antibiotic concentration indicates intermediate sensitivity.

\section{Statistical analysis}

Means and standard deviations were calculated to present continuous variables and proportions were incorporated for categorical variables. Univariate and multivariate logistic regression analyses were performed to evaluate the clinical factors influencing CMP among the patients confirmed with urinary UP infection. All statistical analyses were performed with SPSS version 24.0 (SPSS, Chicago, IL), and $P$ value $\leq 0.05$ was considered as statistically significant.

\section{Results}

\section{Baseline characteristics and clinically significant factors}

Total 276 patients were included in the final analysis and their baseline clinical factors are presented in Table 1. The mean age of the cohort was 42.5 years and none of the patients showed either chronic kidney disease with eGFR $<90 \mathrm{~mL} / \mathrm{min}$ or proteinuria in urine analysis. More than half of the patients (172 patients, $62.3 \%$ ) showed grade $2 \mathrm{MH}$ (11-25 RBCs/HPF). No patient was treated with anticoagulation medication as shown in exclusion criteria (Figure 1). Among the overall MH presenting female patients, 49 patients $(17.8 \%)$ showed urinary infection of UP. Urinary UP serotyping showed the prevalence of seorvar-1, $-3,-6$ and -14 were $24.5 \%, 30.6 \%, 18.4 \%$ and $26.5 \%$, respectively. $53.1 \%$ of urinary UP infected patients had co-presence of pyuria. Moreover, serovar-3 $(42.3 \%)$ and $-6(34.6 \%)$ showed markedly higher rates of pyuria than serovar-1 $(15.4 \%)$ and $-14(7.7 \%)$. Among the urinary UP positive patients, $79.6 \%$ (39 patients) were also positive for vaginal UP infection. For vaginal UP infection, the prevalence of serovars-3 (38.5\%) and -14 (33.4\%) were much higher than the other two serotypes (serovar-1: 17.9\%, serovars-6: 10.2\%). Vaginal UP infection accompanied whitish or clear discharge from vagina and 
serovars-14 showed the highest vaginal discharge rate (61.5\%) compared with other serotypes. Among the whole study cohort, only 22 patients $(8.0 \%)$ had CMP and none of them were positive for UTI with UP serovars-6. In contrast, serovars-3 and -14 infected patients presented similarly high rates of CMP, which were $54.5 \%$ and $41.0 \%$ respectively. $26.4 \%$ (73 patients) of the study cohort were infertile whereas $23.3 \%$ of these infertile patients were positive for UTI with only UP serotype-3 or -14 . The etiologies of infertility for the females, confirmed with UTI by UP serotype-3 (8 patients), were either tubaric or uterine factors. In addition, the female infertile patients positive for urinary UP serotype-14 had their infertility resulted from only tubaric factors.

\section{Antibiotics sensitivity of $U P$}

The antibiotic sensitivity profiles of urinary UP confirmed from the study cohort are presented in Table 3. Regarding susceptibility to quinolones, $71.4 \%$ ( 35 cases) of urinary UP isolates were resistant to ofloxacin whereas $83.7 \%$ (41 cases) were resistant to ciprofloxacin. Serovar-3 of UP showed the highest resistance rate to both ciprofloxacin $(93.3 \%)$ and ofloxacin $(80.0 \%)$. Doxycycline, josamycin and pristinamycin were the most active antibiotics with the lowest rate of resistance $(0.0 \%)$ for treating UP. However, other macrolide antibiotics such as azithromycin, clarithromycin and erythromycin were relatively ineffective for treating UP as the susceptibility to the corresponding antibiotics were $67.3 \%, 77.6 \%$ and $65.3 \%$, respectively. UP serovars-3 showed especially higher resistance rate to these macrolide antibiotics compared with other serotypes (azithromycin: 86.7\%, clarithromycin: $93.3 \%$, erythromycin 93.3\%).

\section{Reinfection of UP during follow up}

All of the patients confirmed with UP infection initially received $100 \mathrm{mg}$ of doxycycline twice a day for 1 week and they were followed up at least for 6-month. The follow up test results for UP infection are presented in Tables 4,5. At 1-month post-initial treatment, all UP serotypes were eradicated and no patient complained of urethral discomfort. However, simultaneous urinary and vaginal reinfection of serovar-3 (5 cases) and serovar-5 (1 case) were confirmed at 3-months post-initial doxycycline therapy. All of the reinfection cases accompanied micturition urethral pain at 3-month follow-up whereas the corresponding urethral discomfort reappeared 9- to 11-week after the initial antibiotics therapy. However, the patients, who were negative for UP reinfection at 3-month follow-up exam, maintained urethral pain-free-state. The 6 reinfection cases were again treated with $100 \mathrm{mg}$ of doxycycline twice a day for 1 week without analyzing antibiotics susceptibility of UP. This booster therapy removed urethral discomforts from the patients within 2 weeks. Despite the second doxycycline therapy, 2 cases of UP reinfection were detected at 6-months post-initial treatment. The two cases consisted of both urinary and vaginal infection by serovars- 3 whereas the other UP serotypes did not present any cases of reinfection at this time. Moreover, these two serovars-3 reinfection cases were associated with micturition urethral pain and they were still sensitive to doxycycline according to antibiotics susceptibility tests.

\section{Factors associated with chronic micturition urethral pain}

The logistic regression analyses evaluating the clinical factors associated with CMP in urinary UP infected patients are presented in Table 6. According to the results, serovars-3 (HR 1.354, $\mathrm{P}$ value 0.018 ) and -14 (HR 1.103, $\mathrm{P}$ value 0.046 ) were significantly associated with CMP in female patients having MH. Serovar-6 and MH grade 3 (26-50 RBCs/HPF) were associated with CMP in univariate analysis, but lost their independent predictor status in multivariate analysis.

\section{Discussion}

Although some previous studies evaluated the prevalence of UP and UU in non-pregnant sexually active symptomatic or asymptomatic women by using multiplex PCR tests, the prevalence has been widely ranged between 3.1-89\% according to different ethnicities and countries (15-18). To our knowledge, there is no study analyzed the infection rate of UP in female patients with $\mathrm{MH}$, and the novelty of this study lies with evaluating the prevalence of UP and UP serotypes for the first time in female patients accompanying MH with/without CMP. The current study presented $17.8 \%$ of females with $\mathrm{MH}$ had urinary tract UP infection. As this study excluded the patients who were simultaneously infected with both UP and other microorganisms such as UU or MG, the actual prevalence of UP might be higher than the corresponding value. According to the current study results, the prevalence of each UP serotype was relatively evenly distributed among the urinary UP infected patients. Thus, there might be no significant differences 
Table 3 Susceptibility of urinary tract infected UP to commonly used antibiotics

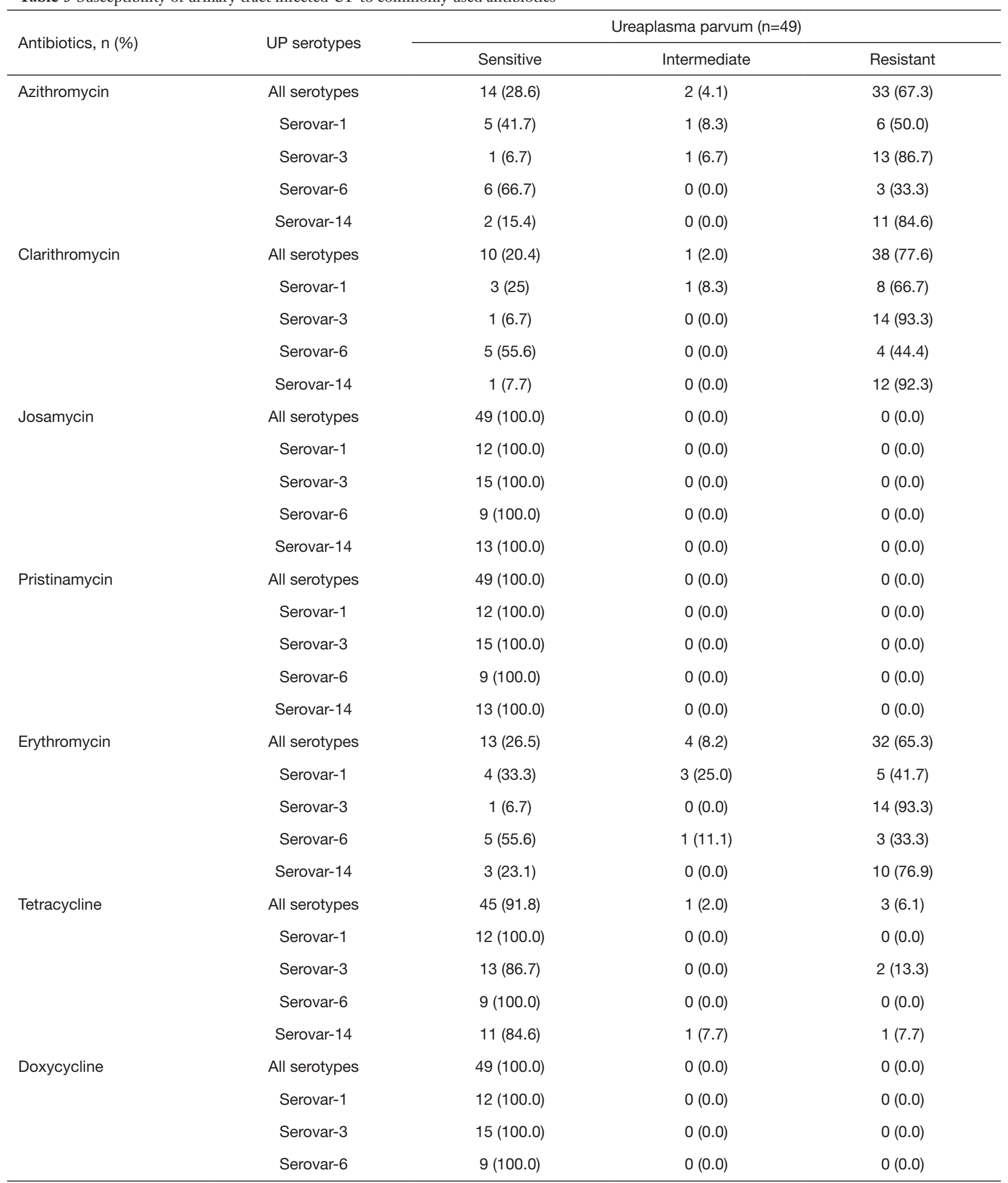

Table 3 (continued) 
Table 3 (continued)

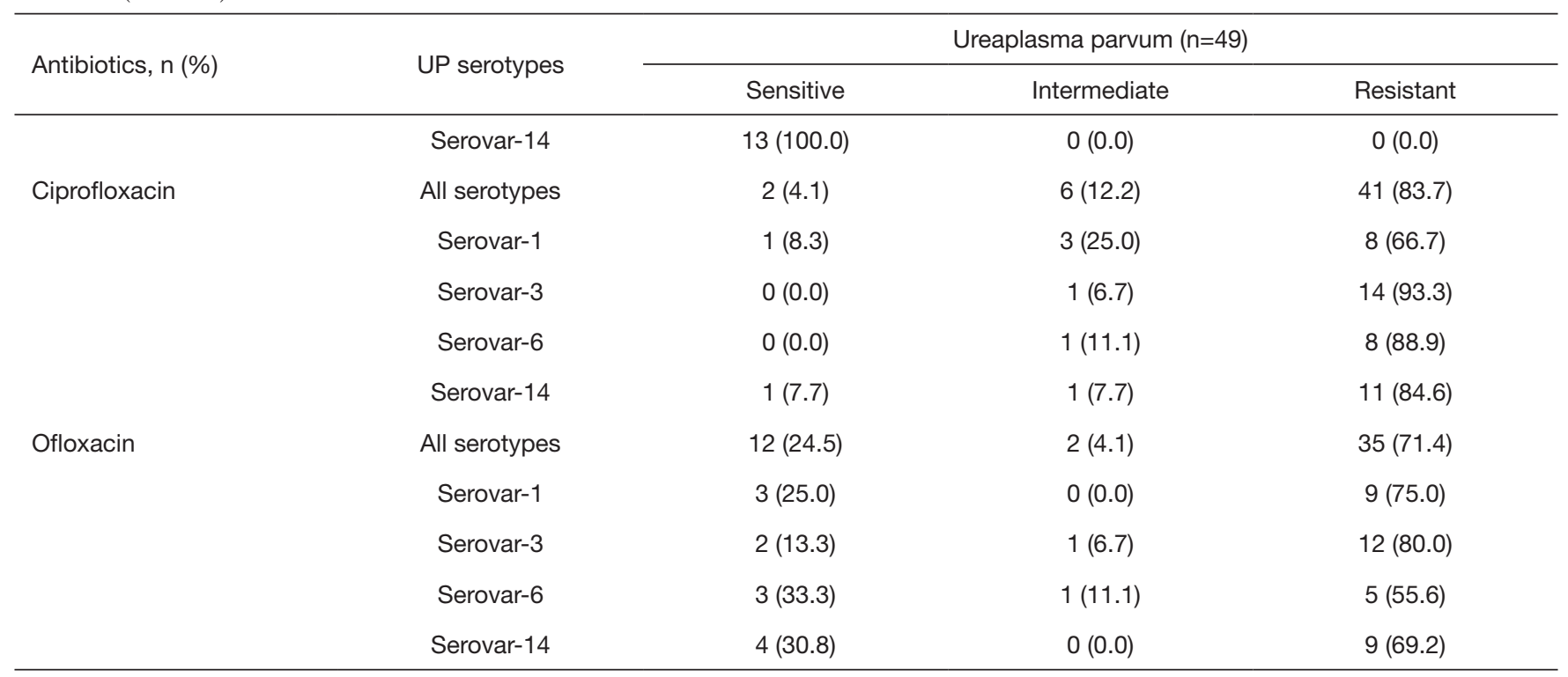

UP, Ureaplasma parvum. Breakpoints of antibiotics sensitivity (mg/L): azithromycin [sensitive $(\mathrm{S}) \leq 0.12 /$ resistant $(\mathrm{R}) \geq 4$ ], clarithromycin [S $\leq 1 /$ $R \geq 4]$, josamycin [S$\leq 2 / R \geq 8$ ], pristinamycin $[R \geq 2]$, erythromycin $[S \leq 1 / R \geq 4]$, tetracycline $[S \leq 4 / R \geq 8]$, doxycycline [S $\leq 4 / R \geq 8]$, ciprofloxacin [S $\leq 1 /$ $R \geq 2]$, ofloxacin $[S \leq 1 / R \geq 4]$.

Table 4 Follow-up UA, vaginal swab and urine PCR for initial urinary UP positive patients $(\mathrm{n}=49)$

\begin{tabular}{|c|c|c|c|}
\hline & 1-month post-initial Tx & 3-month post-initial Tx & 6-month post-initial Tx \\
\hline Present & $0(0.0)$ & $6(12.2)$ & $2(4.1)$ \\
\hline Negative & $49(100.0)$ & $43(87.8)$ & $47(95.9)$ \\
\hline \multicolumn{4}{|c|}{${ }^{\mathrm{F}}$ Micturition urethral pain, $\mathrm{n}(\%)$} \\
\hline Negative & $49(100.0)$ & $43(87.8)$ & $47(95.9)$ \\
\hline \multicolumn{4}{|c|}{ Urinary UP serovars, n (\%) } \\
\hline Serovar-1 & $0(0.0)$ & $0(0.0)$ & $0(0.0)$ \\
\hline Serovar-3 & $0(0.0)$ & $5(10.2)$ & $2(4.1)$ \\
\hline \multicolumn{4}{|c|}{ Vaginal UP infection among urine UP infection, $\mathrm{n}(\%)$} \\
\hline Serovar-1 & $0(0.0)$ & $0(0.0)$ & $0(0.0)$ \\
\hline Serovar-3 & $0(0.0)$ & $5(10.2)$ & $2(4.1)$ \\
\hline Serovar-6 & $0(0.0)$ & $0(0.0)$ & $0(0.0)$ \\
\hline Serovar-14 & $0(0.0)$ & $1(2.0)$ & $0(0.0)$ \\
\hline
\end{tabular}

UA, urine analysis; PCR, polymerase chain reaction; UP, Ureaplasma parvum; Tx, treatment. ${ }^{\mp}$ Only serovar-3/-14 were associated with both recurrent microscopic hematuria and urethral pain at 3-months post-initial treatment. Two patients with voiding pain at 6-months post-initial treatment had UP serovar-3 infection. 
regarding host infectivity between each UP serovar, but further evaluations are needed to confirm it.

A previous study described that UP serovar-3 and -14 are associated with an absence of Lactobacillus species in normal vaginal flora whereas serovar-6 was associated with the presence of Lactobacillus species (19). Other studies

Table 5 Antibiotics susceptibility of two recurrent UP cases at 6-months initial $T_{x}(n=2)$

\begin{tabular}{llll}
\hline & Sensitive & Intermediate & Resistant \\
\hline Azithromycin & $0(0.0)$ & $0(0.0)$ & $2(100.0)$ \\
Clarithromycin & $0(0.0)$ & $0(0.0)$ & $2(100.0)$ \\
Josamycin & $2(100.0)$ & $0(0.0)$ & $0(0.0)$ \\
Pristinamycin & $2(100.0)$ & $0(0.0)$ & $0(0.0)$ \\
Erythromycin & $0(0.0)$ & $0(0.0)$ & $2(100.0)$ \\
Tetracycline & $1(50.0)$ & $1(50.0)$ & $0(0.0)$ \\
Doxycycline & $2(100.0)$ & $0(0.0)$ & $0(0.0)$ \\
Ciprofloxacin & $0(0.0)$ & $0(0.0)$ & $2(100.0)$ \\
Ofloxacin & $0(0.0)$ & $0(0.0)$ & $2(100.0)$ \\
\hline
\end{tabular}

including Campisciano et al. (20) described that serovars-3 was more frequently colonized within the urogenital tract of sexually active females in their reproductive ages compared with other UP serotypes. Our study results were similar but not quite the same to those previous study results. The current study showed that urinary serovar-3 and-14 infections accompanied simultaneous vaginal infection with greater frequencies than serovar-1 and -6 . Furthermore, only serovars- 3 and -14 had recurrent urogenital infections at 3-months after the initial doxycycline therapy. Thus, it could be deduced that UP seorvar-3 and -14 were associated with chronic vaginal colonization, which might subsequently cause recurrent urinary infections. Our study results also presented 1 recurrent infection case by serovars-3 at 6 -months post-initial antibiotics therapy. However, it is still too early to conclude that serovars-3 has significantly higher recurrent infection rate than other serotypes as the size of cohort in the current study was relatively small.

Another point emerging from the present study is related to a strong association of specific UP serotypes with CMP. Our study results presented that $8.0 \%$ of female

Table 6 Univariate and multivariate analysis for predictive factors of chronic micturition urethral pain among urinary UP positive patients

\begin{tabular}{|c|c|c|c|c|c|c|}
\hline & \multicolumn{3}{|c|}{ Univariate } & \multicolumn{3}{|c|}{ Multivariate } \\
\hline \multicolumn{7}{|l|}{ Age } \\
\hline$<61$ years & \multicolumn{3}{|c|}{ Reference } & \multicolumn{3}{|c|}{ Reference } \\
\hline$\geq 61$ years & 0.795 & $0.526-1.902$ & 0.191 & 0.868 & $0.215-2.429$ & 0.372 \\
\hline $4-10$ & \multicolumn{3}{|c|}{ Reference } & \multicolumn{3}{|c|}{ Reference } \\
\hline $11-25$ & 1.103 & $0.323-1.460$ & 0.128 & 1.087 & $0.154-1.845$ & 0.290 \\
\hline $26-50$ & 1.121 & $1.017-1.682$ & 0.027 & 1.025 & $0.983-1.796$ & 0.061 \\
\hline \multicolumn{7}{|c|}{ Presence of DM } \\
\hline \multicolumn{7}{|c|}{ Urinary UP serotypes } \\
\hline Serovar-1 & \multicolumn{3}{|c|}{ Reference } & \multicolumn{3}{|c|}{ Reference } \\
\hline Serovar-3 & 1.637 & $1.356-1.990$ & 0.006 & 1.354 & $1.116-1.892$ & 0.018 \\
\hline Serovar-6 & 0.882 & $0.225-0.906$ & 0.040 & 0.910 & $0.623-1.081$ & 0.089 \\
\hline Serovar-14 & 1.204 & $1.045-1.410$ & 0.035 & 1.117 & $1.031-1.299$ & 0.046 \\
\hline
\end{tabular}

UP, Ureaplasma parvum; HR, hazard ratio; Cl, confidence interval; DM, diabetes mellitus. 
$\mathrm{MH}$ patients complained of CMP indicating $44.8 \%$ of the urinary UP positive patients accompanied chronic voiding urethral pain. Moreover, UP serovar-3 and -14 also showed higher rates of pyuria and CMP presence compared with serovar-1 and -6. Many studies reported UP infection induced urethritis and urethral pain syndrome in female patients, but no meta-analysis or case control studies have undertaken. A study described that $46 \%$ of female patients with urethral pain had Ureaplasma species in their urinary tract (21). Another recent study described that urogenital UP infection effectively results in local host defense such as active secretion of immune substances including lysozyme $(13,22)$. Some other studies, which were based on animal experiments, presented rats infected with UP in bladder having conformational changes in actin-binding-proteins that provokes inflammatory response $(10,23)$. These studies also suggested that the clinical signs and symptoms induced by UP infection are dependent on the host associated factors $(10,23)$. In the current study, multivariate analysis showed that serovar-3 and -14 were significantly associated with CMP in female MH patients. As serovar-3 and -14 accompanied predominantly higher rates of pyuria than the other serotypes in this study, we believe serovar-3 and -14 might have caused more intense local host immune response, which resulted in CMP with greater frequencies. Nonetheless, further study regarding serotype-specific genes and virulence factors inducing CMP is still required to confirm the UP serotype dependent pathogenicity.

Regarding female infertility, the association of UU is well established but the role of UP infection on non-pregnant women is relatively insufficient (24). A previous study demonstrated that UP produces biofilm, which promotes persistent low genital tract infection and it might result in ascending infection to the upper reproductive tract (25). Furthermore, the risk of infertility by Ureaplasma species has been shown to be associated with tubal damages (26). Our study results were similar to those previous studies as $16.3 \%$ (8 patients) of urinary UP infected patients were infertile due to predominantly tubal factor. However, since this study mainly focused on urethral discomfort and $\mathrm{MH}$ induced by urinary UP infection, the pathophysiology of UP related infertility was not further investigated.

Many previous studies evaluated the prevalence of the antibiotic resistance profile of Ureaplasma species but the results were different according to age and country of the subjects (15,27-29). An epidemiological study presented that Ureaplasma species had high levels of resistance for quinolones (90.9\% for ciprofloxacin) and markedly low level of resistance for tetracyclines $(0.0 \%$ for tetracycline and doxycycline) (29). Our results were similar as $6.1 \%$ and $0 \%$ of UP were resistant to tetracycline and doxycycline, respectively. Some macrolides, including josamycin and pristinamycin, were also sensitive to all UP isolates. Although routinely testing and treating UP are still debatable and worldwide proven management guidelines for UP is not available (30), the current study results presented strong association between UP serovar-3/-14 infection and urogenital complications including CMP, MH and female infertility. As recent improvement of molecular tools such as multiplex PCR, has provided easier detection and antibiotics sensitivity measurement for UP (15), we suggest testing for UP and UP serotypes should be performed to the female patients accompanying CMP with/without $\mathrm{MH}$, especially if the patients had medical history of infertility.

As limitations of the study, we must acknowledge that relatively small number of the patients were included in our study. Molecular level evaluations for virulence factors of seorvar-3/-14 were not performed, but we believe further evaluations on the virulent genes are strongly recommended to establish management guideline for UP. Despite the limitations, the current study is still the first research analyzed the role of specific UP serotypes regarding CMP in female patients with $\mathrm{MH}$.

\section{Conclusions}

UP serovars-3 and -14 infections could be significantly associated with CMP and tubal factor infertility in female patients having MH. Doxycycline, josamycin and pristinamycin were effective for treating UP. Serovar-3 showed higher reinfection rate than other serotypes after antibiotics treatment.

\section{Acknowledgments}

Funding: None.

\section{Footnote}

Reporting Checklist: The authors have completed the STROBE reporting checklist. Available at http://dx. doi. org/10. 21037/tau-20-920

Data Sharing Statement: Available at http://dx. doi. org/10. 21037/tau-20-920 
Peer Review File: Available at http://dx.doi.org/10.21037/ tau-20-920

Conflicts of Interest: All authors have completed the ICMJE uniform disclosure form (available at http://dx. doi. org/10.21037/tau-20-920). The authors have no conflicts of interest with any institutions or products.

Ethical Statement: The authors are accountable for all aspects of the work in ensuring that questions related to the accuracy or integrity of any part of the work are appropriately investigated and resolved. All procedures performed in this study were in accordance with the Declaration of Helsinki (as revised in 2013) and approved by the Ethics Committee of the Bundang CHA Hospital, CHA Medical University (registration ID 2020-05-016). All patients enrolled in the study completed the written informed consent form regarding use of their medical records.

Open Access Statement: This is an Open Access article distributed in accordance with the Creative Commons Attribution-NonCommercial-NoDerivs 4.0 International License (CC BY-NC-ND 4.0), which permits the noncommercial replication and distribution of the article with the strict proviso that no changes or edits are made and the original work is properly cited (including links to both the formal publication through the relevant DOI and the license). See: https://creativecommons.org/licenses/by-nc-nd/4.0/.

\section{References}

1. Davis R, Jones JS, Barocas DA, et al. Diagnosis, evaluation and follow-up of asymptomatic microhematuria (AMH) in adults: AUA guideline. American Urological Association Education and Research, Inc., 2012:1-30.

2. Yamagata K, Iseki K, Nitta K, et al. Chronic kidney disease perspectives in Japan and the importance of urinalysis screening. Clin Exp Nephrol 2008;12:1-8.

3. Khadra MH, Pickard RS, Charlton M, et al. A prospective analysis of 1,930 patients with hematuria to evaluate current diagnostic practice. J Urol 2000;163:524-7.

4. Coresh J, Turin TC, Matsushita K, et al. Decline in estimated glomerular filtration rate and subsequent risk of end-stage renal disease and mortality. JAMA 2014;311:2518-31.

5. Singla N, Gayed BA, Bagrodia A, et al. Multi-institutional analysis of renal function outcomes following radical nephroureterectomy and partial ureterectomy for upper tract urothelial carcinoma. Urol Oncol 2015;33:268.e1-7.

6. Deguchi T, Yoshida T, Miyazawa T, et al. Association of Ureaplasma urealyticum (biovar 2) with nongonococcal urethritis. Sex Transm Dis 2004;31:192-5.

7. Yoshida T, Deguchi T, Maeda SI, et al. Quantitative detection of Ureaplasma parvum (biovar 1) and Ureaplasma urealyticum (biovar 2) in urine specimens from men with and without urethritis by real-timepolymerase chain reaction. Sex Transm Dis 2007;34:416-9.

8. Stellrecht KA, Woron AM, Mishrik NG, et al. Comparison of multiplex PCR assay with culture for detection of genital Mycoplasmas. J Clin Microbiol 2004;42:1528-33.

9. Volgmann T, Ohlinger R, Panzig B. Ureaplasma urealyticum: harmless commensal or underestimated enemy of human reproduction? a review. Arch Gynecol Obstet 2005;273:133-9.

10. Allam AB, Alvarez S, Brown MB, et al. Ureaplasma infection alters filamin A dynamics in host cells. BMC Infect Dis 2011;11:101-14.

11. Kacerovský K, Pavlovsky M, Tosner J. Preterm premature rupture of the membranes and genital mycoplasmas. Acta Medica (Hradec Karlove) 2009;52:117-20.

12. Kim M, Kim G, Romero R, et al. Biovar diversity of Ureaplasma urealyticum in amniotic fluid: distribution, intrauterine inflammatory response and pregnancy outcomes. J Perinat Med 2003;31:146-52.

13. Zanotta N, Campisciano C, Morassut S, et al. Emerging role for Ureaplasma parvum serovar 3: Active infection in women with silent high-risk human papilloma virus and in women with idiopathic infertility. J Cell Physiol 2019;234:17905-11.

14. Rittenschober-Böhm J, Waldhoer T, Schulz SM, et al. Vaginal Ureaplasma parvum serovars and spontaneous preterm birth. Am J Obstet Gynecol 2019;220:594. e1-9.

15. Leli C, Mencacci A, Latino MA, et al. Prevalence of cervical colonization by Ureaplasma parvum, Ureaplasma urealyticum, Mycoplasma hominis and Mycoplasma genitalium in childbearing age women by a commercially available multiplex real-time PCR: An Italian observational multicentre study. J Microbiol Immunol Infect 2018;51:220-5.

16. Esen B, Gozalan A, Sevindi DF, et al. Ureaplasma urealyticum: presence among sexually transmitted diseases. Jpn J Infect Dis 2017;70:75-9.

17. Marovt M, Kese D, Kotar T, et al. Ureaplasma parvum and Ureaplasma urealyticum detected with the same 
frequency among women with and without symptoms of urogenital tract infection. Eur J Clin Microbiol Infect Dis 2015;34:1237-45.

18. Lobão TN, Campos GB, Selis NN, et al. Ureaplasma urealyticum and $\mathrm{U}$. parvum in sexually active women attending public health clinics in Brazil. Epidemiol Infect 2017;145:2341-51.

19. De Francesco MA, Negrini R, Pinsi G, et al. Detection of Ureaplasma biovars and polymerase chain reactionbased subtyping of Ureaplasma parvum in women with or without symptoms of genital infections. Eur J Clin Microbiol Infect Dis 2009;28:641-6.

20. Campisciano G, Florian F, D'Eustacchio A, et al. Subclinical alteration of the cervical-vaginal microbiome in women with idiopathic infertility. J Cell Physiol 2017;232:1681-8.

21. Kyndel A, Elmer C, Kallman O, et al. Mycoplasmataceae colonizations in women with urethral pain syndrome: A case-control study. J Low Genit Tract Dis 2016;20:272-4.

22. Glaser K, Silwedel C, Fehrholz M, et al. Ureaplasma species differentially modulate pro- and anti-Inflammatory cytokine responses in newborn and adult human monocytes pushing the state toward pro-inflammation. Front Cell Infect Microbiol 2017;7:484.

23. Reyes L, Reinhard M, Brown MB. Different inflammatory responses are associated with Ureaplasma parvum-induced UTI and urolith formation, BMC Infect Dis 2009;9:9.

24. Hunjak B, Sabol I, Vojnović G, et al. Ureaplasma urealyticum and Ureaplasma parvum in women of

Cite this article as: Kim MS, Lee DH, Kim TJ, Oh JJ, Rhee SR, Park DS, Yu YD. The role of Ureaplasma parvum serovar-3 or serovar-14 infection in female patients with chronic micturition urethral pain and recurrent microscopic hematuria. Transl Androl Urol 2021;10(1):96-108. doi: 10.21037/tau-20-920 reproductive age. Arch Gynecol Obstet 2014;289:407-12.

25. Kasprzykowska U, Elias J, Elias M, et al. Colonization of the lower urogenital tract with Ureaplasma parvum can cause asymptomatic infection of the upper reproductive system in women: a preliminary study. Arch Gynecol Obstet 2014;289:1129-34.

26. Piscopo RC, Guimarães RV, Ueno J, et al. Increased prevalence of endocervical Mycoplasma and Ureaplasma colonization in infertile women with tubal factor. JBRA Assist Reprod 2020;24:152-7.

27. Kotrotsiou T, Exindari M, Diza E, et al. Prevalence and antimicrobial susceptibility of Ureaplasma urealyticum in asymptomatic women in Northern Greece. Hippokratia 2013;17:319-21.

28. Kweon OJ, Lim YK, Oh SM, et al. Prevalence and antimicrobial susceptibility of Mycoplasma hominis, Ureaplasma urealyticum and Ureaplasma parvum in individuals with or without symptoms of genitourinary infections. Lab Med Online 2016;6:79-87.

29. Song T, Huang J, Liu Z, et al. Antibiotic susceptibilities and genetic variations in macrolide resistance genes of Ureaplasma spp. isolated in China. New Microbiol 2019;42:225-7.

30. Horner P, Donders G, Cusini M, et al. Should we be testing for urogenital Mycoplasma hominis, Ureaplasma parvum and Ureaplasma urealyticum in men and women? - a position statement from the European STI Guidelines Editorial Board. J Eur Acad Dermatol Venereol 2018;32:1845-51. 